\title{
Structures of vector control of n-phase motor drives based on generalized Clarke transformation
}

\author{
M. JANASZEK* \\ Electrotechnical Institute, 28 Pożaryskiego St., 04-703 Warsaw, Poland
}

\begin{abstract}
The paper presents vector control structures for n-phase AC motors derived from generalized Clarke transformation. In contrast to known works, where authors operate on generalized formulas of the Clarke transformation, in this work, only a number of actually used phases, of mostly industrial purposes, are considered $(\mathrm{n}=5,6,2 \times 3)$. This allows to perform control calculations in stationary orthogonal coordinates $\mathrm{a} \beta$ or rotating dq. There are implementations of different control strategies: ROC (rotor-oriented control) or FOC (field-oriented control). Next, the paper presents the novel concept of a voltage modulator designed for multi-phase drive systems. Operation of the modulator is based on the extended Clarke transformation for multiphase systems. A mathematical model of the multiphase voltage modulator, operating in open-loop and closed-loop current control has been presented. Some selected oscillograms of voltage and current waveform, which illustrate properties of proposed control structures have been presented.
\end{abstract}

Key words: electric drive, electric motor, vector control of multiphase system, Clarke transformation.

\section{Introduction}

At present, the most widely used electric drives in industry are 3-phase AC induction and PMSM motors. Power amplifiers are transistors bridge circuits. In the last ten years in the literature describing the electrical drive systems, there are more and more publications related to $n$-phase systems, i.e. where the number of motor phases $n>3$ [1-4]. Due to the number of phases $\mathrm{n}>3$, such motors cannot be powered directly from a 3-phase grid, but must be supplied with power by transistor converters on the number of branches equal to the number of motor phases. In electrical engineering, for the analysis of waveforms of different physical quantities, e.g. voltage, current, flux, in multiphase systems the vector notation is used $[5,6]$.

Space vectors are defined based on physical quantities (voltages, currents, flux linkages) in the individual phases of a motor [7]. Space vectors are written in a stationary n-phase coordinate system for multiphase drive, fixed with the stator. Torque and motor speed are regulated by a control system implementing adequate feedback. Most often, the drive is built with a primary loop speed control. As a subordinated torque or current control, loops are used. It is convenient to write regulatory procedures in the stationary coordinate system $\alpha \beta$ or rotating dq synchronized with a rotor or stator flux. Control strategies like: rotor-oriented control (ROC) or field-oriented control (FOC) have been realized, basing on coordinate transformations. For 3-phase systems, the space vector written for stationary systems phase $\mathbf{X}_{\mathrm{ABC}}$ is transformed into orthogonal $\mathbf{X}_{\alpha \beta}$ through the Clarke transformation [8]. As the paper addresses n-phase systems built from a number of phases $n>3$, one should define the transformation of the n-phase to orthogonal transformation, which is a general-

*e-mail: m.janaszek@iel.waw.pl ization of Clarke transformation for systems with a number of phases $n>3$. In contrast to known works $[9,10]$ where authors operate on generalized formulas of the Clarke transformation, in this work, only a number of actually used phases, of mostly industrial purposes, are considered $(n=5,6,2 \times 3)$.

\section{Why multiphase drive systems?}

In the last years, more and more often there appear works devoted to multiphase drives. Most of them refer to drives with induction motors, but published there are also descriptions of drives with permanent, magnet-synchronous motors. Many works refer to drives with 5-phase, but also with 6-phase motors, and dual 3-phase motors. A work on drives with multiphase motors justifies the advantages of such motors, compared to 3-phase motors:

- With the same nominal values of power and supply voltage, the nominal values of phase currents for an n-phase motor are reduced, as compared to a 3-phase one.

- In transistor power converters, components of a smaller permissible collector current can be applied. This problem is important in high-power drives used in traction, metallurgy, mining transport, high-power pump drives, fans, and compressors [11, 12].

- In n-phase systems, the angle between the phases of the motor is smaller. When using direct torque control method, it is possible to accurately determine the position of the rotor.

- In n-phase motors, there is a decrease in the amplitude of fluctuations of the torque, and an increase in their frequency (for a specified angular velocity of the rotor) [13].

- By increasing the number of phases, the drive offers a greater robustness. It is possible to work with a damaged single phase in the motor or branch in the inverter [14]. 
At the same time, n-phase motors, as compared to the 3-phase, are characterized by:

- Larger complexity of the mechanical structure.

- Complex phenomena related to the conversion of electric energy into mechanical energy.

- N-phase motors must be supplied with n-phase power converters, so that the number of phases of the motor and the inverter are the same.

The control circuit of an n-phase drive must be adapted to the number of phases, while it can use the control strategies as those in conventional drives. Classical vector control method is executed most frequently in the cascade structures, which play a decisive role in fast current vector loop.

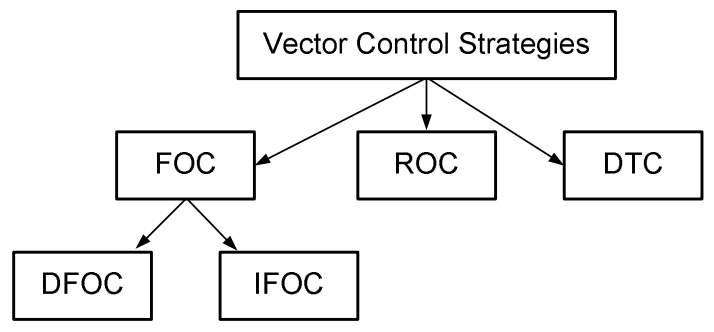

Fig. 1. Division of basic vector control strategies used in n-phase drives

- FOC - the field oriented control, involving the synchronization of the stator current vector with respect to the flux position. It is the famous control strategy for an induction motor. The principle of FOC is based on an analogy to the separately-excited DC motor. Field-oriented-controlled machines need two variables as input references: the torque and the flux. FOC consists of controlling the stator currents represented by a vector. The space vector can be represented in another reference frame, with only two orthogonal axis $\alpha, \beta$ (Clarke transformation). Next, the Park transformation modifies a two-phase orthogonal system $\alpha, \beta$ in the $\mathrm{d}$, $q$ rotating reference frame. These transformations require a rotor flux angle $\theta$. Depending on calculations of this angle, two different kinds of field-oriented control methods may be considered. If the flux angle $\theta$ is estimated from motor signals and parameters, this is called direct FOC, and if it is calculated from speed and slip frequency, it is called indirect FOC (Fig. 2).

- $\mathrm{ROC}$ - rotor oriented control, involving the synchronization of the stator current vector with respect to the rotor position. It is a less popular control strategy. In a synchronous machine, the rotor speed is equal to the rotor flux speed. Then, the rotor flux position $\theta$ is directly measured by a position sensor.

These control methods require a transformation of space vectors from the n-phase coordinates to the orthogonal coordinate system.

- DTC - direct torque control strategy. The n-phase inverter produces $\mathrm{m}=2^{\mathrm{n}}$ voltage vector, including two zero vectors. Thus, for multiphase systems, the number of active vectors is:

$$
\begin{array}{ll}
\text { 3-phase } & \mathrm{m}=2^{3}-2=8-2=6, \\
\text { 4-phase } & \mathrm{m}=2^{4}-2=16-2=14, \\
\text { 5-phase } & \mathrm{m}=2^{5}-2=32-2=30, \\
\text { 6-phase } & \mathrm{m}=2^{6}-2=64-2=62 .
\end{array}
$$

In order to avoid calculations in case of a large number of vectors used in a drive, the n-phase modulator is proposed. Its operation will involve converting vector quantities from the n-phase coordinates to orthogonal coordinates, and vice versa. In the literature [2,8], drive systems with a multi-hysteresis current regulator are described (Fig. 2). Usually, the control system applies the speed control loop. The speed controller generates a reference value component $i_{\text {qref }}$ of the current vector. The reference component $i_{d r e f}$ of the current vector is set directly. Reference current components $i_{\text {dref }}, i_{\text {qref }}$ are converted into components $i_{\alpha}, i_{\beta}$ in $\alpha, \beta$ system through Park transformation.

Reference phase currents are compared with the measured values of the phase currents in the hysteresis controller. Such a control structure needs to operate on n-phase values.

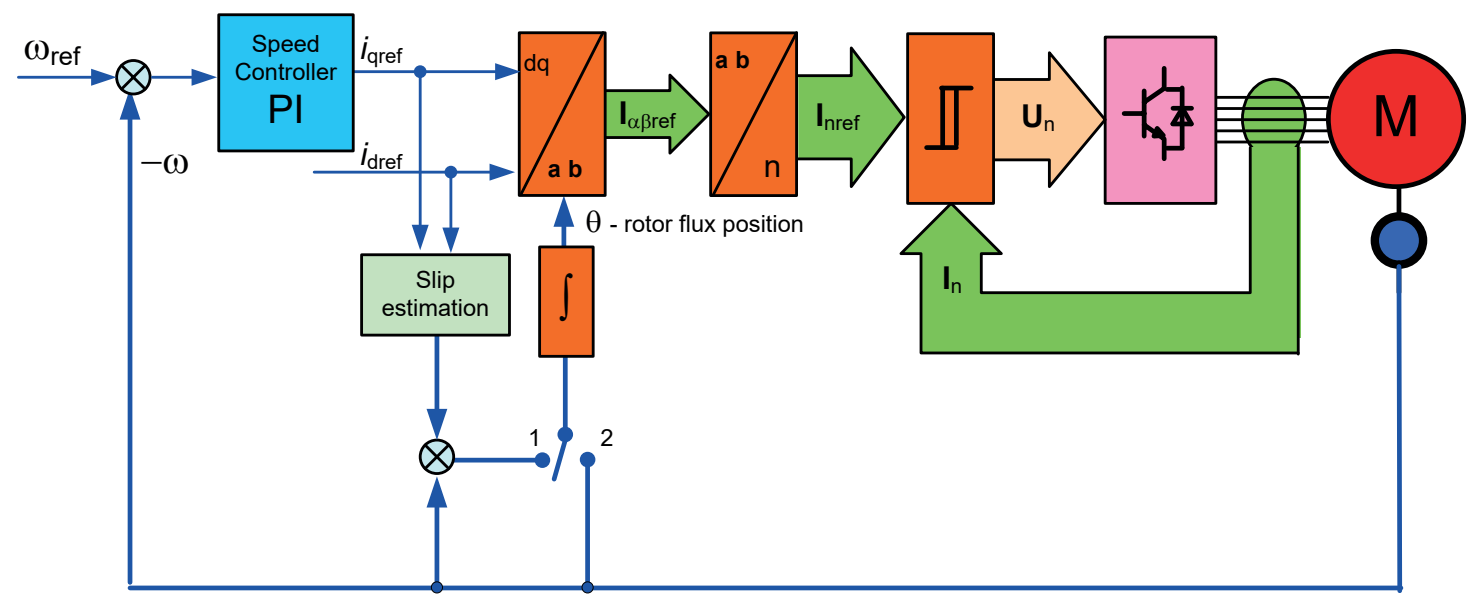

Fig. 2. Control scheme with hysteresis current controller for multiphase drive: 1 - Indirect FOC for the induction motor, 2 - ROC for PMSM 


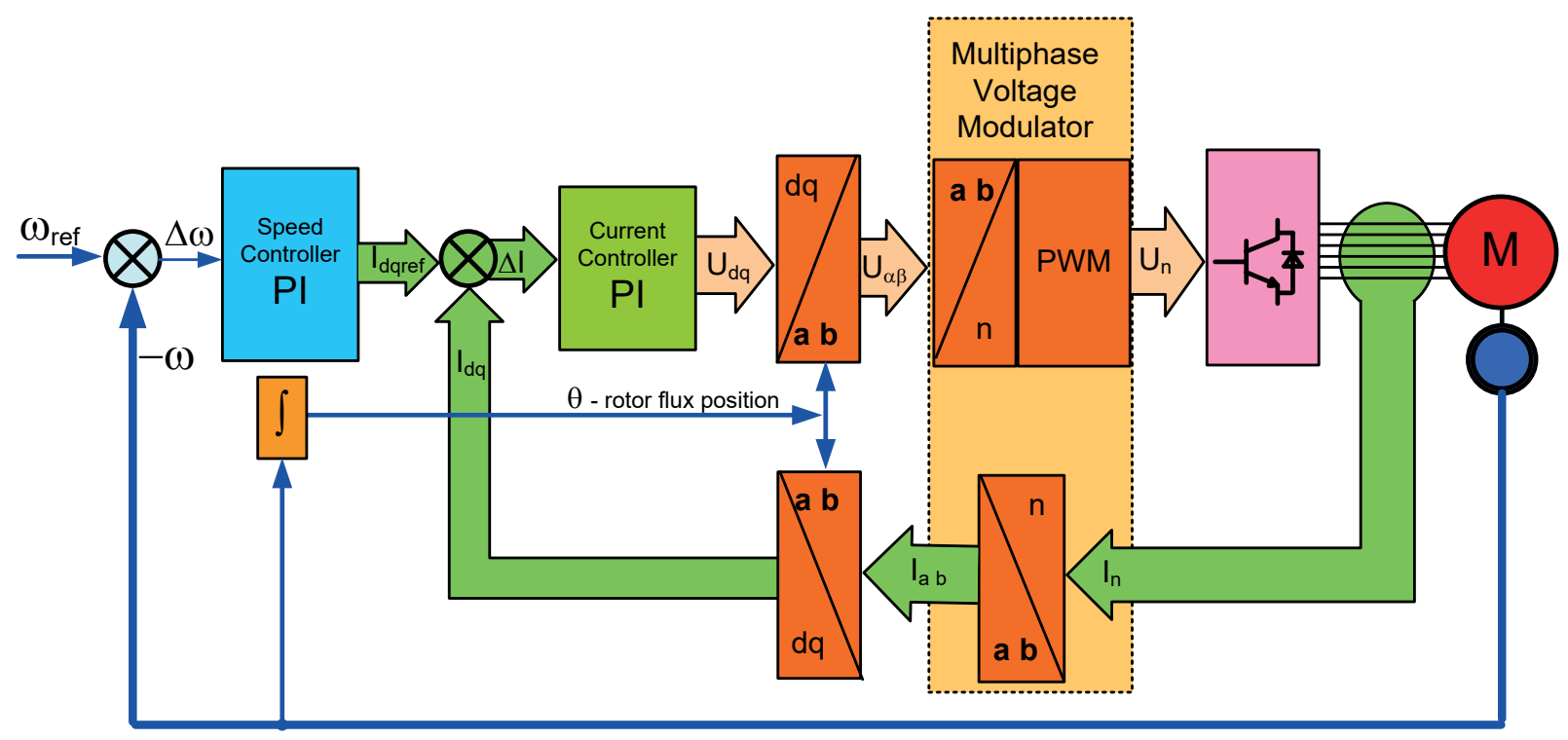

Fig. 3. ROC scheme with linear PI current controller for multiphase drive

This paper proposes the application of a control structure with n-phase voltage modulator (Fig. 3). This allows the use of current regulators - PI and PWM circuit. This allows for obtaining uniform current waveforms. Using a superior speed control loop and a speed controller PI, a reference component $i_{\text {qref }}$ of the current vector is generated. Reference component $i_{\text {dref }}$ is adjusted directly.

The objective of the paper is to simplify the control algorithms in a fast-current control loop. In the calculations, the elements of transformation matrices $\mathbf{T}_{\alpha \beta / \mathrm{n}}(16)$ and $\mathbf{T}_{\mathrm{n} / \alpha \beta}(23)$ can be written in the form of simple fractions (Tables 1,3). This can be considered a contribution to the simplification of the control strategy of multiphase systems.

\section{Assumptions for an n-phase system}

For further discussion, we accept the following assumptions (Fig. 4):

1. The system consists of $\mathrm{n}$ phases, and we will consider in detail the systems for $n=3,4,5,6,2 \times 3$.

2. The $n$-phase stator winding is connected in Star, with an isolated neutral.

3. The angle between successive phases is: $\theta_{n}=\frac{2 \pi}{n}$, which operates for the following systems:

$$
\begin{array}{ll}
\text { 3-phase } & \theta_{3}=\frac{2 \pi}{3}=120^{\circ}, \\
\text { 4-phase } & \theta_{4}=\frac{2 \pi}{4}=\frac{\pi}{2}=90^{\circ}, \\
\text { 5-phase } & \theta_{5}=\frac{2 \pi}{5}=72^{\circ}, \\
\text { 6-phase } & \theta_{6}=\frac{2 \pi}{6}=\frac{\pi}{3}=60^{\circ}, \\
\text { dual 3-phase } & \theta_{2 \times 3}=\frac{2 \pi}{12}=\frac{\pi}{6}=30^{\circ} .
\end{array}
$$

4. The vector in the phase coordinates is written in the form of:

$$
\mathbf{X}_{\mathrm{n}}=\left[\begin{array}{c}
x_{1}(t) \\
x_{2}(t) \\
\vdots \\
x_{\mathrm{n}}(t)
\end{array}\right],
$$

where: $x_{1}(t), x_{2}(t), \ldots, x_{\mathrm{n}}(t)$ are the instantaneous values of the phase quantities.

5. The sum of the instantaneous values of the phase quantities equals to zero:

$$
\sum_{\mathrm{k}=1}^{\mathrm{k}=\mathrm{n}} x_{\mathrm{k}}(t)=0
$$

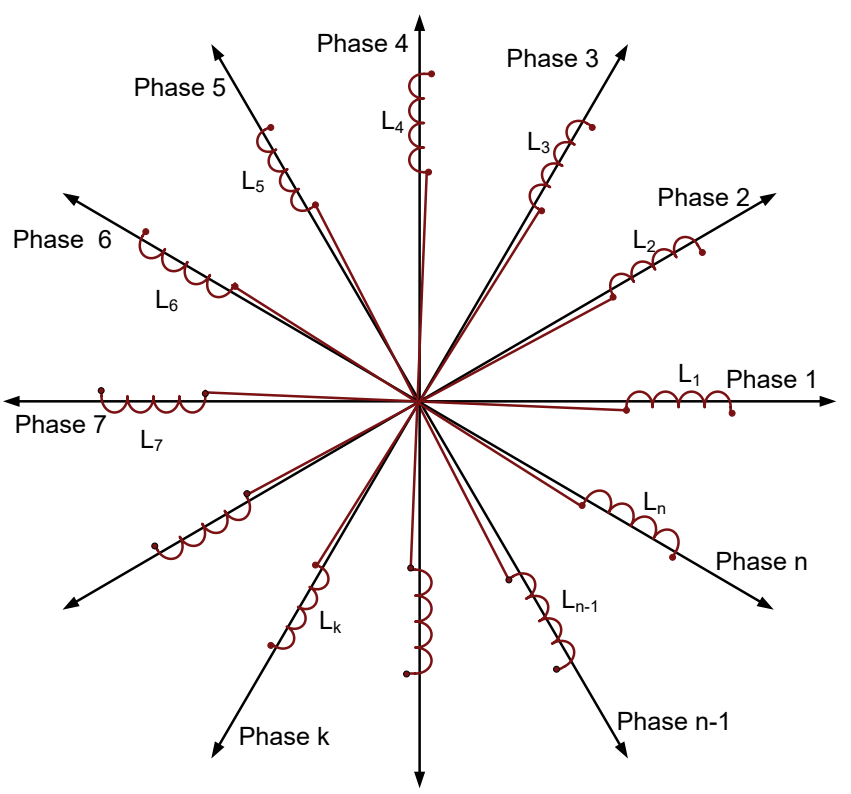

Fig. 4. Simplified diagram of the stator windings of $n-$ phase motor 


\section{Multiphase modulator in an open loop system}

Input signals of the modulator are:

- components $u_{\text {dref }}, u_{\text {qref }}$ of the voltage vector:

$$
\mathbf{U}_{\mathrm{dq}}=\left[\begin{array}{l}
u_{\text {dref }} \\
u_{\text {qref }}
\end{array}\right]=|\mathbf{U}| \cdot\left[\begin{array}{c}
\cos (\alpha) \\
\sin (\alpha)
\end{array}\right]
$$

where: $|\mathbf{U}|$ - module of voltage vector

$$
\alpha \text { - the initial angle of the voltage vector }
$$

- pulsation $\omega$ :

Voltage vector components $u_{\text {dref }}, u_{\text {qref }}$ are converted into components $u_{\alpha}, u_{\beta}$ in stationary coordinates system by the Park transformation:

$$
\begin{gathered}
\mathbf{U}_{\alpha \beta}=\left[\begin{array}{l}
u_{\alpha} \\
u_{\beta}
\end{array}\right]=|\mathbf{U}| \cdot\left[\begin{array}{cc}
\cos (\omega t) & -\sin (\omega t) \\
\sin (\omega t) & \cos (\omega t)
\end{array}\right] \cdot\left[\begin{array}{c}
\cos (\alpha) \\
\sin (\alpha)
\end{array}\right], \\
\mathbf{U}_{\alpha \beta}=\left[\begin{array}{l}
u_{\alpha} \\
u_{\beta}
\end{array}\right]=|\mathbf{U}| \cdot\left[\begin{array}{c}
\cos (\omega t+\alpha) \\
\sin (\omega t+\alpha)
\end{array}\right] .
\end{gathered}
$$

Next, the transformation of the voltage vector $\mathbf{U}_{\alpha \beta}$ into the n-phase stationary coordinates system is performed. The transformation is based on multiplication of the vector $\mathbf{U}_{\alpha \beta}$ by a matrix $\mathbf{T}_{\alpha \beta / \mathrm{n}}$ :

$$
\mathbf{U}_{\mathrm{n}}=\mathbf{T}_{\alpha \beta / \mathrm{n}} \cdot \mathbf{U}_{\alpha \beta} .
$$

The transformation matrix is:

$$
\mathbf{T}_{\alpha \beta / n}=\left[\begin{array}{cc}
1 & \multicolumn{1}{c}{0} \\
\cos \left(\frac{2 \pi}{\mathrm{n}}\right) & -\sin \left(\frac{2 \pi}{\mathrm{n}}\right) \\
\cos \left(\frac{4 \pi}{\mathrm{n}}\right) & -\sin \left(\frac{4 \pi}{\mathrm{n}}\right) \\
: & : \\
\cos \left(\frac{2 \mathrm{k} \pi}{\mathrm{n}}\right) & -\sin \left(\frac{2 \mathrm{k} \pi}{\mathrm{n}}\right) \\
\vdots & : \\
\cos \left(\left(\frac{2(n-1) \pi}{\mathrm{n}}\right)\right. & -\sin \left(\frac{2(n-1) \pi}{\mathrm{n}}\right)
\end{array}\right]
$$

For the number of phases $n=3,4,5,6$, the matrix elements can be written in the form of a fraction (Table 1).
As a result of the transformation, the voltage vector $\mathbf{U}_{n}$ in n-phase stationary coordinate system is obtained:

$$
\mathbf{U}_{\mathrm{n}}=\left[\begin{array}{l}
u_{1} \\
u_{2} \\
u_{3} \\
\vdots \\
u_{\mathrm{k}} \\
\vdots \\
u_{\mathrm{n}}
\end{array}\right]=|\mathbf{U}| \cdot\left[\begin{array}{c}
\cos (\omega t+\alpha) \\
\cos \left(\omega t+\alpha+\frac{2 \pi}{\mathrm{n}}\right) \\
\cos \left(\omega t+\alpha+\frac{4 \pi}{\mathrm{n}}\right) \\
\cdots \\
\cos \left(\omega t+\alpha+\frac{2 \mathrm{k} \pi}{\mathrm{n}}\right) . \\
\cdots \cdots \\
\cos \left(\omega t+\alpha+\frac{2(\mathrm{n}-1) \pi}{\mathrm{n}}\right)
\end{array}\right],
$$

where: $\omega$-value of pulsation

$\alpha$ - the initial angle of the voltage vector

$\frac{2 \mathrm{k} \pi}{\mathrm{n}}-$ the angle of the phase $\mathrm{k}$

$|\mathbf{U}|$ - module of voltage vector

If the output voltages are applied to n-phase resistive-inductive circuit, connected in Star, and being a load of the inverter, then the current vector $I_{n}$ is obtained:

$$
\mathbf{I}_{\mathrm{n}}=\left[\begin{array}{l}
i_{1} \\
i_{2} \\
\vdots \\
i_{\mathrm{k}} \\
\vdots \\
i_{\mathrm{n}}
\end{array}\right]=|\mathrm{|}| \cdot\left[\begin{array}{c}
\cos (\omega t+\beta) \\
\cos \left(\omega t+\beta+\frac{2 \pi}{\mathrm{n}}\right) \\
\ldots \ldots \\
\cos \left(\omega t+\beta+\frac{2 \mathrm{k} \pi}{\mathrm{n}}\right) \\
\cdots \cdots \\
\cos \left(\omega t+\beta+\frac{2(\mathrm{n}-1) \pi}{\mathrm{n}}\right)
\end{array}\right] .
$$

Table 1

Matrices $T_{\alpha \beta / n}$ transform vector $\mathbf{U}_{\alpha \beta}$ from an orthogonal system of coordinates $\boldsymbol{\alpha} \boldsymbol{\beta}$ to the n-phase stationary coordinates $\mathbf{U}_{n}$

\begin{tabular}{|l|c|}
\hline $\mathrm{n}$ & $\mathbf{T}_{\alpha \beta / \mathrm{n}}$ \\
\hline 3 & {$\left[\begin{array}{cc}1 & 0 \\
\cos \left(\frac{2 \pi}{3}\right) & -\sin \left(\frac{2 \pi}{3}\right) \\
\cos \left(\frac{4 \pi}{3}\right) & -\sin \left(\frac{4 \pi}{3}\right)\end{array}\right]=\left[\begin{array}{cc}1 & 0 \\
-\frac{1}{2} & -\frac{\sqrt{3}}{2} \\
-\frac{1}{2} & \frac{\sqrt{3}}{2}\end{array}\right]$} \\
\hline 4 & {$\left[\begin{array}{cc}1 & 0 \\
\cos \left(\frac{\pi}{2}\right) & -\sin \left(\frac{\pi}{2}\right) \\
\cos (\pi) & -\sin (\pi) \\
\cos \left(\frac{3 \pi}{2}\right) & -\sin \left(\frac{3 \pi}{2}\right)\end{array}\right]=\left[\begin{array}{cc}1 & 0 \\
0 & -1 \\
-1 & 0 \\
0 & 1\end{array}\right]$} \\
\hline
\end{tabular}

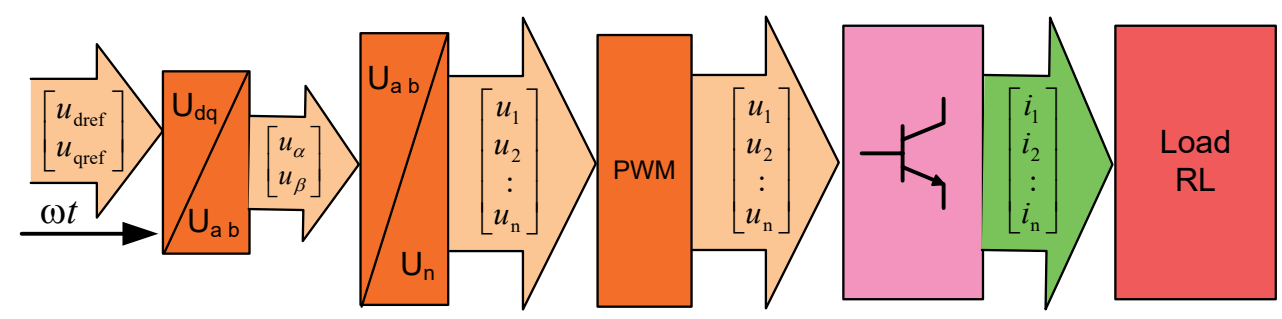

Fig. 5. The scheme of the n-phase modulator in open loop 


\begin{tabular}{|l|c|}
\hline $\mathrm{n}$ & $\mathbf{T}_{\alpha \beta / \mathrm{n}}$ \\
\hline 5 & {$\left[\begin{array}{cc}1 & 0 \\
\cos \left(\frac{2 \pi}{5}\right) & -\sin \left(\frac{2 \pi}{5}\right) \\
\cos \left(\frac{4 \pi}{5}\right) & -\sin \left(\frac{4 \pi}{5}\right) \\
\cos \left(\frac{6 \pi}{5}\right) & -\sin \left(\frac{6 \pi}{5}\right) \\
\cos \left(\frac{8 \pi}{5}\right) & -\sin \left(\frac{8 \pi}{5}\right)\end{array}\right]=\left[\begin{array}{cc}1 & 0 \\
\frac{\sqrt{5}-1}{4} & -\frac{\sqrt{10+2 \sqrt{5}}}{4} \\
-\frac{\sqrt{5}+1}{4} & -\frac{\sqrt{10-2 \sqrt{5}}}{4} \\
-\frac{\sqrt{5}+1}{4} & \frac{\sqrt{10-2 \sqrt{5}}}{4} \\
\frac{\sqrt{5}-1}{4} & \frac{\sqrt{10+2 \sqrt{5}}}{4}\end{array}\right]$} \\
\hline 6 & {$\left[\begin{array}{cc}1 & 0 \\
\cos \left(\frac{\pi}{3}\right) & -\sin \left(\frac{\pi}{3}\right) \\
\cos \left(\frac{2 \pi}{3}\right) & -\sin \left(\frac{2 \pi}{3}\right) \\
\cos (\pi) & -\sin (\pi) \\
\cos \left(\frac{4 \pi}{3}\right) & -\sin \left(\frac{4 \pi}{3}\right) \\
\cos \left(\frac{5 \pi}{3}\right) & -\sin \left(\frac{5 \pi}{3}\right)\end{array}\right]=\left[\begin{array}{cc}1 & 0 \\
\frac{1}{2} & -\frac{\sqrt{3}}{2} \\
-\frac{1}{2} & -\frac{\sqrt{3}}{2} \\
-1 & 0 \\
-\frac{1}{2} & \frac{\sqrt{3}}{2} \\
\frac{1}{2} & \frac{\sqrt{3}}{2}\end{array}\right]$} \\
\hline $2 \times 3$ & {$\left[\begin{array}{cc}1 & 0 \\
\cos \left(\frac{2 \pi}{3}\right) & -\sin \left(\frac{\pi}{3}\right) \\
\cos \left(\frac{4 \pi}{3}\right) & -\sin \left(\frac{2 \pi}{3}\right) \\
\cos \left(\frac{\pi}{6}\right) & -\sin \left(\frac{\pi}{6}\right) \\
\cos \left(\frac{5 \pi}{6}\right) & -\sin \left(\frac{5 \pi}{6}\right) \\
\cos \left(\frac{9 \pi}{6}\right) & -\sin \left(\frac{9 \pi}{6}\right)\end{array}\right]=\left[\begin{array}{cc}1 & 0 \\
-\frac{1}{2} & -\frac{\sqrt{3}}{2} \\
-\frac{1}{2} & \frac{\sqrt{3}}{2} \\
\frac{\sqrt{3}}{2} & -\frac{1}{2} \\
-\frac{\sqrt{3}}{2} & -\frac{1}{2} \\
0 & 1\end{array}\right]$} \\
\hline
\end{tabular}

4.1. Examples of the results of simulation. A mathematical model of an n-phase voltage modulator operating in open loop system has been built. The following circuit parameters have been used (Table 2). As input signals are:

- Pulsation of voltage modulator:

$$
\omega=314 \frac{\mathrm{rad}}{\mathrm{s}},
$$

- Voltage vector components:

$$
\begin{aligned}
& u_{\text {dref }}(t)=100 \cdot \sin (62,8 t), \\
& u_{\text {qref }}(t)=-100 \cdot \sin (62,8 t) .
\end{aligned}
$$

Table 2

Parameters of the simulated circuit

\begin{tabular}{|l|l|}
\hline \multicolumn{1}{|c|}{ Parameter } & \multicolumn{1}{c|}{ Value } \\
\hline Resistance of phase load & $\mathrm{R}_{\mathrm{F}}=1 \Omega$ \\
\hline Inductance of phase load & $\mathrm{L}_{\mathrm{F}}=0.01 \mathrm{H}$ \\
\hline Intermediate circuit voltage & $\mathrm{U}_{\mathrm{DC}}=560 \mathrm{~V}$ \\
\hline PWM carrier frequency & $\mathrm{f}_{\mathrm{PWM}}=10 \mathrm{kHz}$ \\
\hline Pulsation of voltage & $\omega=314 \frac{\mathrm{rad}}{\mathrm{s}}$ \\
\hline
\end{tabular}

Module of the reference voltage vector is:

$$
\left|\mathrm{U}_{\mathrm{S}}\right|=\sqrt{u_{\mathrm{dref}}^{2}+u_{\mathrm{qref}}^{2}}=100 \cdot \sqrt{2} \cdot|\sin (62,8 \cdot t)| .
$$

Calculations of voltage and current waveforms for the modulator from Fig. 6 were performed for the 3-phase, 4-phase, 5-phase, 6-phase, and dual 3-phase systems (Fig. 7).

a)
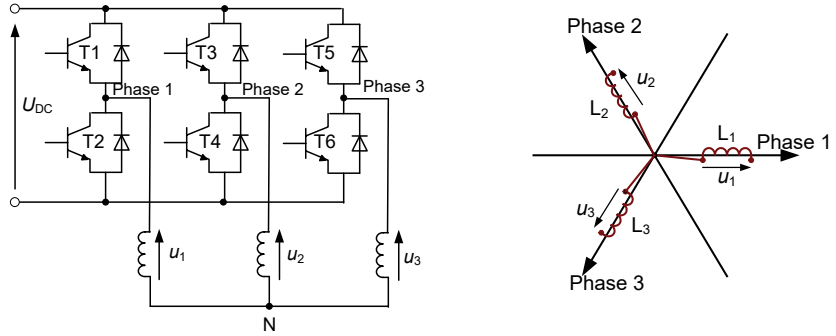

b)
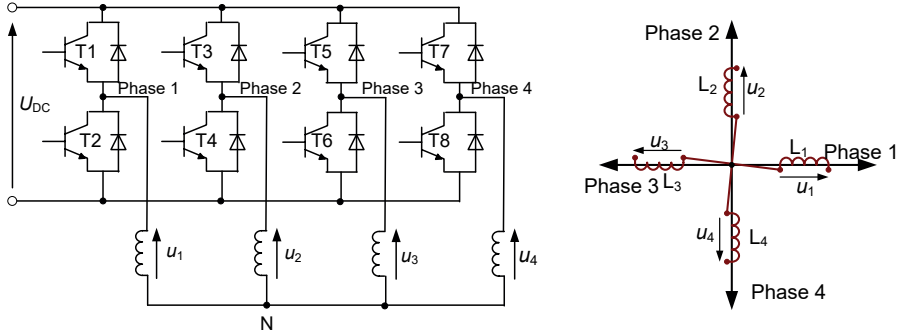

c)
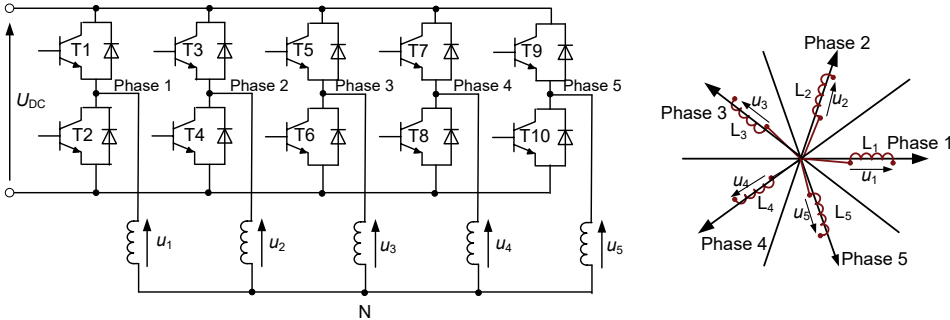

d)
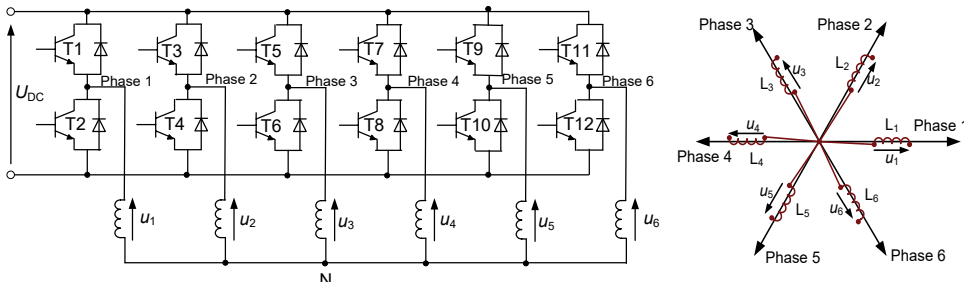

e)
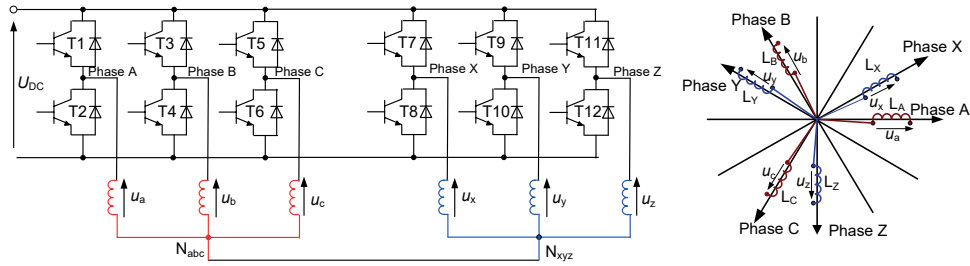

Fig. 6. Scheme of the converter and allocation of the system phases: a) 3-phase, b) 4-phase, c) 5-phase, d) 6-phase, e) dual 3-phase 
a)

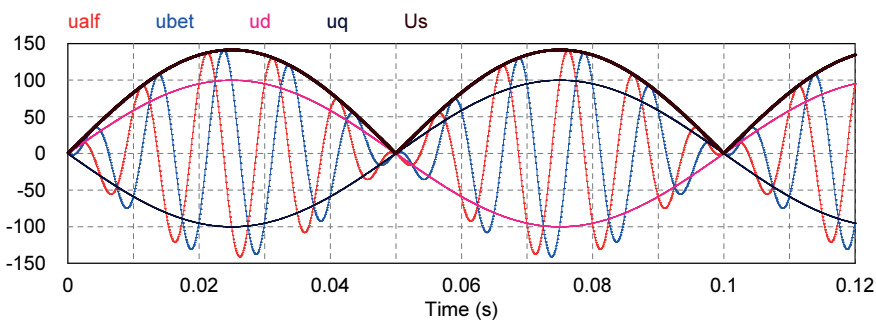

c)

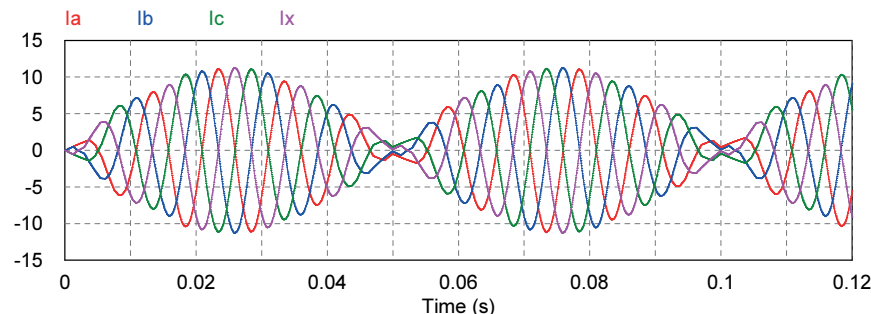

e)

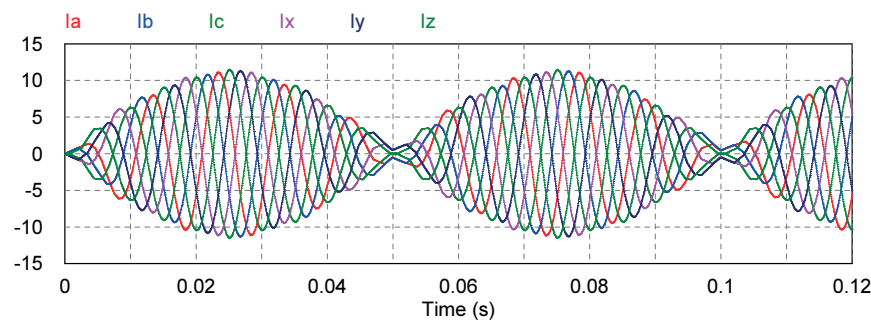

b)

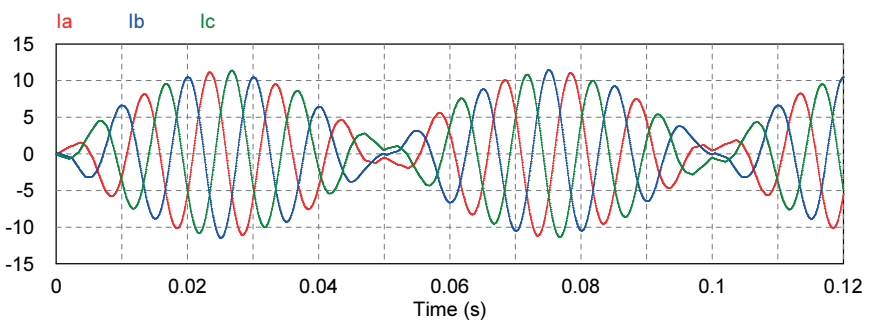

d)

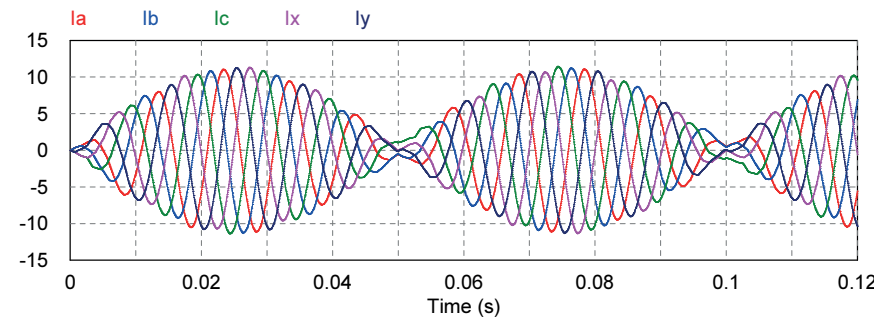

f)

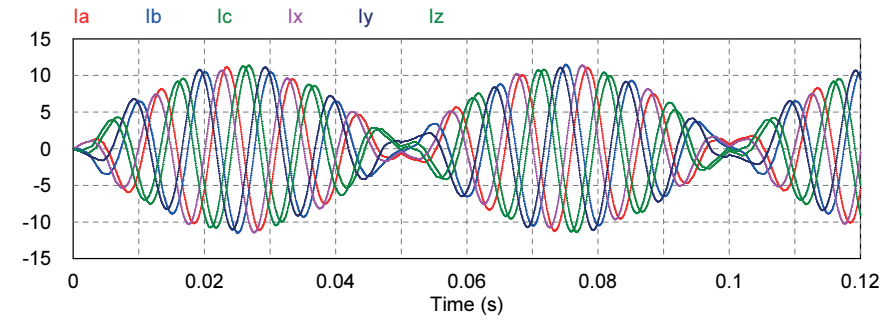

Fig. 7. Simulated voltage and current waveforms for the modulator from shown in Fig. 6: a) reference voltage signals $u_{\mathrm{d}}, u_{\mathrm{q}}$ and $u_{\alpha}, u_{\beta}$, and module of reference voltage vector $U_{S}$. Output phases currents for n-phase circuit: b) 3-phase, c) 4-phase, d) 5-phase, e) 6-phase, f) dual 3-phase

\section{Multiphase modulator in closed current loop}

Operation of the modulator in a closed current control loop requires:

- Measurement of phase currents $i_{1}, i_{2}, \ldots i_{\mathrm{n}}$ defining the current vector $I_{n}(18)$,

- Transformation of current vector $I_{n}$ to the form $I_{\alpha \beta}$ in coordinate system $\alpha, \beta(23,24)$,

- Transformation current vector $\mathbf{I}_{\alpha \beta}$ to the form $\mathbf{I}_{\mathrm{dq}}$ in coordinate system dq (25-27).

Transformation of the current vector $\mathbf{I}_{\mathrm{n}}$ from the $\mathrm{n}$-phase stationary coordinates into the orthogonal stationary coordinates $\alpha, \beta$ is performed with the matrix:

$$
\mathbf{T}_{\mathrm{n} / \alpha \beta}=\frac{2}{\mathrm{n}} \cdot\left[\begin{array}{ccccc}
1 & \cos \left(\frac{2 \pi}{\mathrm{n}}\right) & \cos \left(\frac{4 \pi}{\mathrm{n}}\right) & \ldots \ldots & \cos \left(\frac{2(\mathrm{n}-1) \pi}{\mathrm{n}}\right) \\
0 & -\sin \left(\frac{2 \pi}{\mathrm{n}}\right) & -\sin \left(\frac{4 \pi}{\mathrm{n}}\right) & \ldots \ldots & -\sin \left(\left(\frac{2(\mathrm{n}-1) \pi}{\mathrm{n}}\right)\right.
\end{array}\right] \text {. }
$$

The transformation based on the multiplication of the current vector $\mathbf{I}_{\mathrm{n}}$ by a matrix $\mathbf{T}_{\mathrm{n} / \alpha \beta}$ :

$$
\mathbf{I}_{\alpha \beta}=\mathbf{T}_{\mathrm{n} / \alpha \beta} \cdot \mathbf{I}_{\mathrm{n}} .
$$

For the number of phases $n=3,4,5,6$, the elements of matrix $\mathbf{T}_{\mathrm{n} / \alpha \beta}$ (23) can be written in the form of fractions (Table 2). As a result of the transformation, the current vector $I_{\alpha \beta}$ in orthogonal coordinates $\alpha \beta$ is obtained:

$$
\mathbf{I}_{\alpha \beta}=\left[\begin{array}{c}
i_{\alpha} \\
i_{\beta}
\end{array}\right]=|\boldsymbol{I}| \cdot\left[\begin{array}{c}
\cos (\omega t+\beta) \\
\sin (\omega t+\beta)
\end{array}\right] .
$$

Current vector components $i_{\alpha}, i_{\beta}$ are converted into components $i_{\mathrm{d}}, i_{\mathrm{q}}$ in system dq through Park transformation:

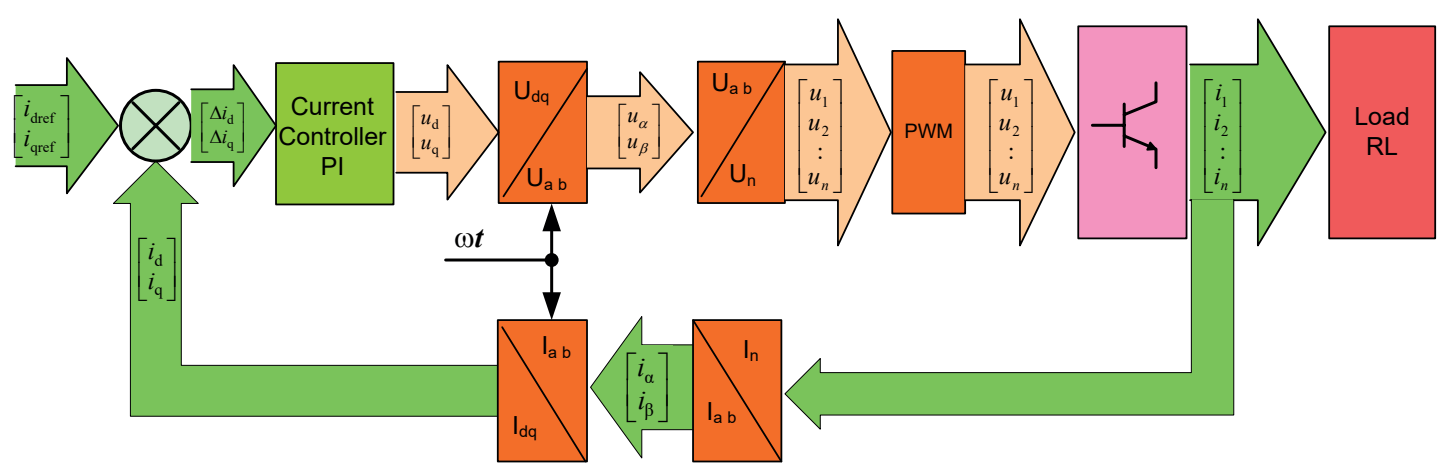

Fig. 8. The scheme of n-phase modulator in closed current loop 


$$
\begin{gathered}
\mathbf{I}_{\mathrm{dq}}=\left[\begin{array}{l}
i_{\mathrm{d}} \\
i_{\mathrm{q}}
\end{array}\right]=\mid \mathrm{I} \cdot\left[\begin{array}{cc}
\cos (\omega t) & \sin (\omega t) \\
-\sin (\omega t) & \cos (\omega t)
\end{array}\right] \cdot\left[\begin{array}{c}
\cos (\omega t+\beta) \\
\sin (\omega t+\beta)
\end{array}\right], \\
\mathbf{I}_{\mathrm{dq}}=\left[\begin{array}{l}
i_{\mathrm{d}} \\
i_{\mathrm{q}}
\end{array}\right]=\mid \boldsymbol{|} \cdot\left[\begin{array}{l}
\cos (\beta) \\
\sin (\beta)
\end{array}\right] .
\end{gathered}
$$

Current vector $\mathbf{I}_{\mathrm{dq}}$ is compared with a reference current vector $\mathbf{I}_{\text {dqref }}$. Current control error is delivered to the current regulator PI. At the output of the current regulator, the reference voltage vector $\mathbf{U}_{\mathrm{dq}}$ has been obtained. Transformations of voltage vectors are performed as described in Section 4.

Table 3

Matrices $\mathbf{T}_{\mathrm{n} / \alpha \beta}$ transformation of vector $\mathbf{U}_{\mathrm{n}}$ from n-phase stationary

\begin{tabular}{|c|c|}
\hline $\mathrm{n}$ & $T_{n / \alpha \beta}$ \\
\hline 3 & $\frac{2}{3} \cdot\left[\begin{array}{ccc}1 & \cos \left(\frac{2 \pi}{3}\right) & \cos \left(\frac{4 \pi}{3}\right) \\
0 & -\sin \left(\frac{2 \pi}{3}\right) & -\sin \left(\frac{4 \pi}{3}\right)\end{array}\right]=\frac{2}{3} \cdot\left[\begin{array}{ccc}1 & -\frac{1}{2} & -\frac{1}{2} \\
0 & -\frac{\sqrt{3}}{2} & \frac{\sqrt{3}}{2}\end{array}\right]$ \\
\hline 4 & $\frac{1}{2} \cdot\left[\begin{array}{cccc}1 & \cos \left(\frac{\pi}{2}\right) & \cos (\pi) & \cos \left(\frac{3 \pi}{2}\right) \\
0 & -\sin \left(\frac{\pi}{2}\right) & -\sin (\pi) & -\sin \left(\frac{3 \pi}{2}\right)\end{array}\right]=\frac{1}{2} \cdot\left[\begin{array}{cccc}1 & 0 & -1 & 0 \\
0 & -1 & 0 & 1\end{array}\right]$ \\
\hline 5 & 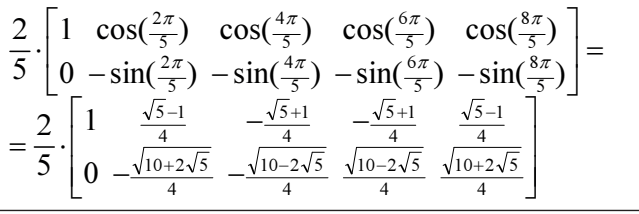 \\
\hline 6 & 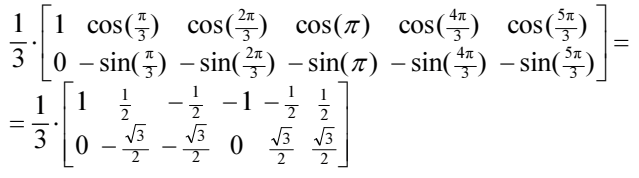 \\
\hline $2 \times 3$ & 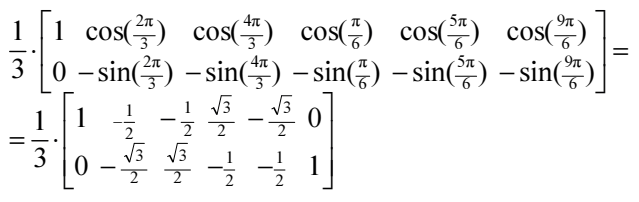 \\
\hline
\end{tabular}
coordinates to orthogonal stationary coordinates $\boldsymbol{\alpha} \boldsymbol{\beta}$

5.1 Examples of the results of simulation. A mathematical model of an $n$-phase voltage modulator operating in a current control loop has been built. The following circuit parameters have been used (Table 2). Input signals were:

- Pulsation of voltage modulator:

$$
\omega=314 \frac{\mathrm{rad}}{\mathrm{s}} \text {, }
$$

- current vector components:

$$
\begin{aligned}
& i_{\text {dref }}(t)=10 \cdot \sin (62,8 t), \\
& i_{\text {qref }}(t)=-10 \cdot \sin (62,8 t) .
\end{aligned}
$$

Module of reference current vector:

$$
\left|\mathrm{I}_{\mathrm{S}}\right|=\sqrt{i_{\text {dref }}^{2}+i_{\text {qref }}^{2}}=10 \cdot \sqrt{2} \cdot|\sin (62,8 \cdot t)| .
$$

Calculations of voltage and current waveforms for the modulator from Fig. 7 were performed for: 3-phase, 4-phase, 5-phase, 6-phase, and dual 3-phase systems (Fig. 9). a)

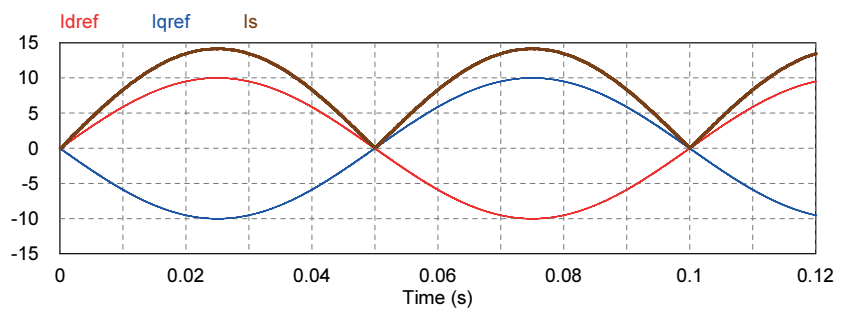

b)
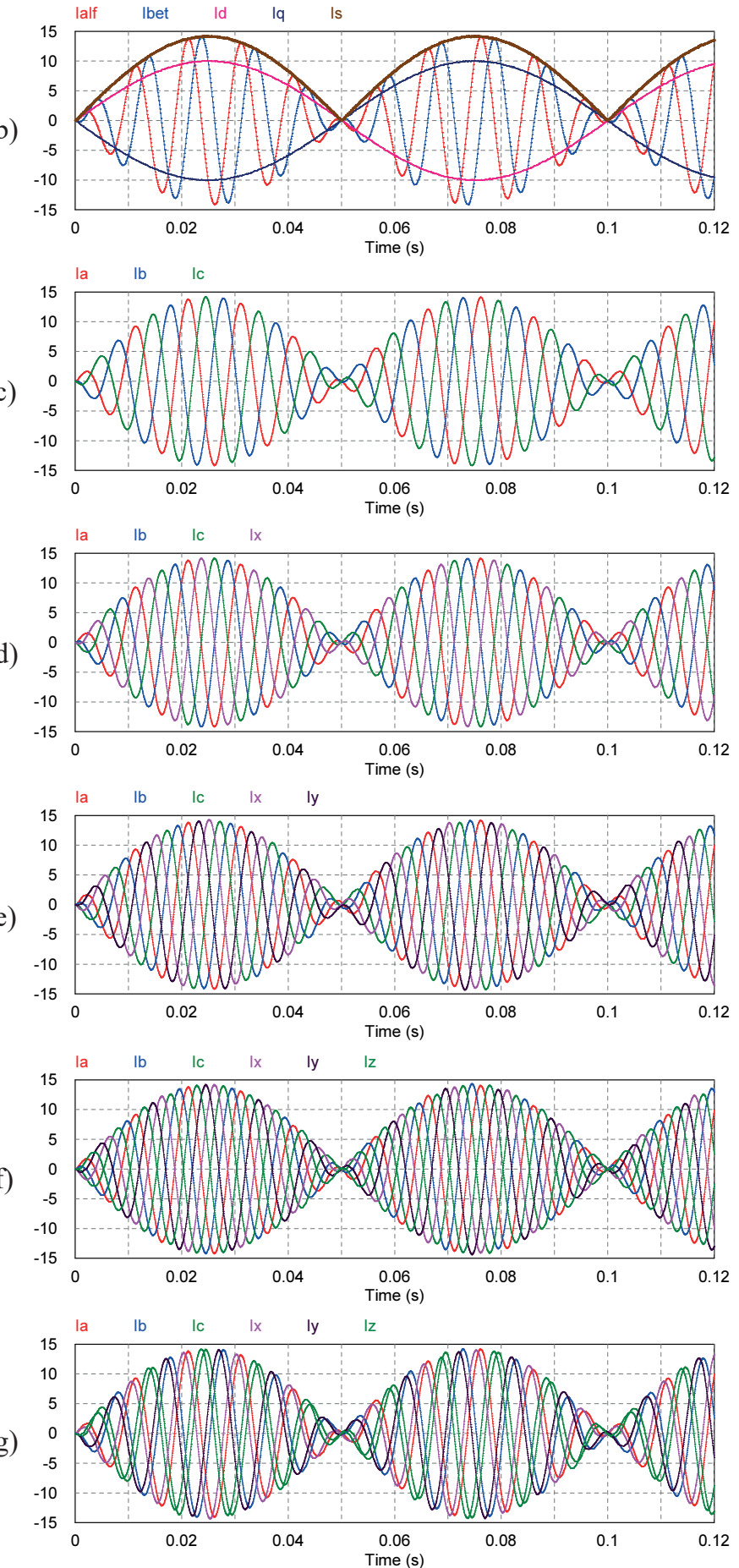

Fig. 9. Simulated current waveforms for the modulator from Fig. 7: a) reference current signals $i_{\text {dref, }} i_{\text {qref }}$ and module of reference current vector $\mathrm{I}_{\mathrm{S}}, \mathrm{b}$ ) output current signals $i_{\mathrm{d}}, i_{\mathrm{q}}$ and $i_{\alpha}, i_{\beta}$ in orthogonal system. Output phase current for n-phase circuit: c) 3-phase, d) 4-phase, e) 5-phase, f) 6-phase, and g) dual 3-phase 


\section{Conclusions}

In this paper, a generalized Clarke transformation and its implementation for an n-phase, inverter-fed AC motor drives have been performed. The generalized Clarke transformation allows to transform a space vector and its mathematical symbol in the n-phase system to the orthogonal system:

- The transformation of vector $\mathbf{X}_{\mathrm{n}}$ from n-phase stationary coordinate system into an orthogonal coordinate system $\boldsymbol{\alpha}$, $\boldsymbol{\beta}$ is carried out by matrix $\mathbf{T}_{\mathrm{n} / \alpha \beta}$. This transformation concerns the vectors of current $\mathbf{I}_{n}$ or voltage $\mathbf{U}_{n}$.

- The inverse transformation, i.e. conversion from vector $\mathbf{X}_{\alpha \beta}$ to vector $\mathbf{X}_{n}$, is conducted by means of matrix $\mathbf{T}_{\alpha \beta / \mathrm{n}}$. This transformation applies the voltage vector $\mathbf{U}_{\mathrm{n}}$ and gives an n-number of control signals for n-phase transistor inverter. Application of generalized Clarke transformation allows to perform calculations in orthogonal coordinate systems - stationary $\boldsymbol{\alpha}, \boldsymbol{\beta}$ or rotating $\mathrm{d}, \mathrm{q}-$ and implement different control strategies: ROC - rotor oriented control or FOC - field oriented control, for multiphase drives.

\section{REFERENCES}

[1] B. Jakubiec, "The drive of electric vehicle with multi-phase permanent magnet synchronous motor", Przeglad Elektrotechniki 91 (12), ISSN 0033-2097, (2015).

[2] M. Rolak, H.S. Che, and M. Malinowski, "Modelling and fault-tolerant control of 5-phase induction machine", Bull. Pol. Ac.: Tech. 63 (4), (2015).

[3] E. Levi, "Multiphase electric machines for variable-speed applications", IEEE Trans. on Ind. El 55 (5), (2008).
[4] L. Chang and J. Muszynski, "Design of a 5-phase permanent magnet brushless DC motor for automobiles", IEEE 58th Vehicular Technology Conference 2003-Fall, (2003).

[5] M. Janaszek, "New method of direct reactive energy and torque control for permanent magnet synchronous motor", Bull. Pol. Ac.: Tech. 54 (3), (2006).

[6] M. Janaszek, "Experimental drive with direct torque control of permanent magnet synchronous motor", Archives of Electrical Engineering 50 (1), 73-92 (2001).

[7] M.P. Kazmierkowski, R. Krishnan, and F. Blaabjerg, "Control in power electronics: Selected problems”, Academic Press, San Diego, 2002.

[8] W.C. Duesterhoeft, M.W. Schulz, E. Clarke, "Determination of instantaneous currents and voltages by means of alpha, beta, and zero components", AIEE Transactions 70, 1248-1255 (1951).

[9] E. Levi, "Multiphase AC machines", pp. 3-31 in 3-1, The Industrial Electronics Handbook: Power electronics and Motor Drives, CRC Press, 2011.

[10] E. Levi, "FOC: Field oriented control", pp. 24-32 in 24-1. The Industrial Electronics Handbook: Power electronics and Motor Drives, CRC Press, 2011.

[11] W. Figna, K. Pieńkowski: "Vector control of induction motor with dual stator winding", Electrical Machines - Transaction Journal 86, (2010).

[12] J. Listwan and K. Pieńkowski: "Analysis of vector control of multi-phase induction motor", Electrical Machines - Transaction Journal 3, (2014).

[13] Y. Ren and Z.Q. Zhu: "Reduction of both harmonic current and torque ripple for dual three-phase permanent-magnet synchronous machine using modified switching-table-based direct torque control", IEEE Trans. on Ind. El. 62 (11), (2015).

[14] A. Kontarcek, P. Bajec, and M. Nemec, "Cost-effective threephase PMSM drive tolerant to open-phase fault", IEEE Trans. on Ind. El. 62 (11), (2015). 